\title{
Association of C-Reactive Protein and Microalbuminuria (from the National Health and Nutrition Examination Surveys, 1999 to 2004)
}

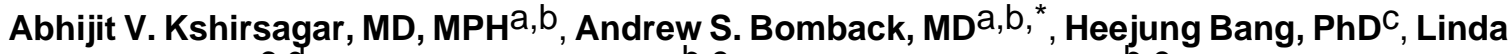
M. Gerber, PhD $^{\mathrm{C}}$, , Suma Vupputuri, PhD ${ }^{b}, \mathrm{e}$, David A. Shoham, $\mathrm{PhD}^{\mathrm{b}, \mathrm{e}}$, Madhu Mazumdar, PhD $^{C}$, Christie M. Ballantyne, MD ${ }^{f}$, James J. Paparello, MDg, and Philip J. Klemmer, MDa,b

${ }^{a}$ Division of Nephrology and Hypertension, School of Medicine, University of North Carolina, USA

bUniversity of North Carolina Kidney Center, Chapel Hill, North Carolina

'Division of Biostatistics and Epidemiology, Department of Public Health, Weill Medical College of Cornell University, New York, New York, USA

dDivision of Nephrology and Hypertension, Weill Medical College of Cornell University, New York, New York, USA

eDepartment of Epidemiology, School of Public Health, University of North Carolina, USA

eDepartment of Medicine, Weill Medical College of Cornell University, New York, New York, USA

fSection of Atherosclerosis and Lipoprotein Research, Department of Medicine, Baylor College of Medicine, and Center for Cardiovascular Disease Prevention, Methodist DeBakey Heart Center, Houston, Texas

gDivision of Nephrology and Hypertension, Feinberg School of Medicine, Northwestern University, Chicago, Illinois
\end{abstract}

\section{Abstract}

Chronic kidney disease and cardiovascular disease share many risk factors. Injury to the vascular endothelium, measured by elevated levels of serum C-reactive protein (CRP), may play a role in kidney and cardiovascular disease. We therefore examined the association of CRP with microalbuminuria, a marker of early kidney injury. We conducted a cross-sectional analysis of a nationally representative, population-based survey. Weighted multiple logistic regression was used to study the association between CRP and microalbuminuria, adjusting for well-known risk factors. CRP was analyzed by a continuous variable and two categorized variables using quartiles and clinically recommended cutpoints. CRP concentration was positively associated with microalbuminuria. In the multivariate model, a one unit (in milligrams per liter) increase in CRP concentration was associated with a $2 \%$ increased odds of microalbuminuria (odds ratio $1.02,95 \%$ confidence interval $[\mathrm{CI}] 1.01$ to $1.02, \mathrm{p}=0.0003$ ). When CRP concentrations were stratified by clinically recommended cutpoints, compared with persons with CRP concentrations $<1 \mathrm{mg} / \mathrm{dl}$, persons with CRP concentrations between 1 and $3 \mathrm{mg} / \mathrm{L}$ and $>3 \mathrm{mg} / \mathrm{L}$ were 1.15 times $(95 \%$ CI 0.94 to 1.42 ) and 1.33 times (95\% CI 1.08 to 1.65 ) more likely to have microalbuminuria, respectively. In subgroup analyses, the strength of association was comparable or stronger. In conclusion, elevated CRP levels were associated with microalbuminuria in a large, nationally representative data set. Vascular inflammation, as measured by CRP, may be a common contributor to early heart and kidney disease.

(C) 2008 Elsevier Inc. All rights reserved.

*Corresponding author: Tel: 919-966-2561; fax: 919-966-4251. abomback@unch.unc.edu (A. Bomback). 
Chronic kidney disease and cardiovascular disease share many risk factors. The similarities in the pathogenesis of cardiovascular and chronic kidney disease suggest that vascular inflammation may have a role in kidney dysfunction. We postulated that C-reactive protein (CRP) is positively associated with microalbuminuria, an easily measured and widely used marker of early kidney injury. Although preliminary supportive evidence exists, the studies are limited by significant methodologic issues including small sample sizes, ${ }^{1-} 5$ the presence of confounding variables, 6 lack of generalizability, 7 and the use of indirect comparisons. $8^{-}$

${ }^{10} \mathrm{We}$ therefore examined the association of CRP and microalbuminuria in a large, diverse data set compiled from the National Health and Nutrition Examination Surveys (NHANES) 1999 through 2004.

\section{Methods}

NHANES are national surveys conducted since 1971 by the National Center for Health Statistics of the Centers for Disease Control and Prevention (CDC). Participants in NHANES are identified through a complex, multistage clustering sample design of the civilian, noninstitutionalized population. Certain under-represented populations, such as older people, racial-ethnic minorities, and low income families, were oversampled. We combined data from 3 independent surveys, available on a public domain website (http://www.cdc.gov/nchs/nhanes.htm), for a total of 6 years: NHANES 1999 to 2000, 2001 to 2002 , and 2003 to 2004 . For our analysis, we restricted the NHANES population to the adult population of men and women aged $\geq 20$ years.

NHANES used trained personnel to ascertain medical and health information from participants by direct interview, examination, and blood samples. We chose comprehensive sociodemographic and clinical variables as potential confounders of the association between CRP and microalbuminuria based on the literature. These included demographic factors (age, gender, race, education), family history of diabetes and hypertension, and personal health history (cholesterol, blood pressure, diabetes mellitus, body mass index [BMI], smoking status, and glomerular filtration rate) (see Table 1 for a complete list).

For the study end point, we first generated a urinary albumin-to-creatinine ratio and then defined microalbuminuria as a ratio between 30 and $300 \mathrm{mg} / \mathrm{g}$. Participants with ratios >300 $\mathrm{mg} / \mathrm{g}$ (i.e., with macroalbuminuria) were excluded from the primary analysis. Various characteristics between persons with microalbuminuria and persons without microalbuminuria were compared. Unadjusted means along with standard errors for continuous variables and proportions for categorical variables were calculated by case status. The differences were tested by $t$ test or Wald chi-square tests, suited for complex survey design. ${ }^{11}$ Key demographic factors (i.e., age, race, and gender) were adjusted for the comparison of all characteristics except when the demographic factors themselves were compared. In addition, we computed parametric (Pearson's) and nonparametric rank-based (Spearman's) correlation coefficients between CRP and important covariates.

We fitted a minimally adjusted model, adjusting for only key demographic factors, and a fully adjusted model, adjusting for a comprehensive set of traditional risk factors. To build a multivariate model, we used the backward elimination technique including all of the covariates listed in Table 1 in the initial model. From the initial model, the variable with the largest $p$ value was removed 1 at a time until we reached the final parsimonious model with only significant predictors $(\mathrm{p} \leq 0.05)$. When a parsimonious set of predictors was established, we retested whether any of the removed variables gained statistical significance when they were reentered into the model to ensure that no variables were deleted erroneously in this sequential process. In addition, variables (i.e., anthropometric measures in this case) based on our a priori knowledge were also added to the model regardless of their statistical significance. In the final 
model, we assured that highly correlated variables were not entered together to avoid multicollinearity. Model simplicity is also important to prevent overadjustment. ${ }^{12}$ For similar or competing variables (e.g., BMI vs waist circumference, systolic vs diastolic blood pressure, education vs income, serum creatinine vs glomerular filtration rate), we considered the relative significance of each variable quantitatively and qualitatively (e.g., missing data proportion and acceptability in clinical and practical settings).

We repeated regression analysis using continuous covariates and categorical covariates separately to investigate potentially useful clinical thresholds for elevated CRP levels. We adopted 2 categorizations: one is based on quartiles and the other is based on the cutpoints recommended by the CDC and the American Heart Association (AHA) ${ }^{13}$ In both analyses, age and BMI were treated as continuous variables to minimize residual confounding. From regression analyses, odds ratios (ORs) were estimated together with the corresponding 95\% confidence intervals (CIs) to quantify the direction and magnitude of associations between each predictor and the study outcome, controlling other variables.

In addition we performed several sensitivity analyses. First, we repeated the analysis for nondiabetic participants to address the over-representation of diabetics among participants with microalbuminuria. Second, we repeated the analysis using gender-specific definitions of microalbuminuria ( $\geq 17 \mathrm{mg} /$ day for men and $\geq 25 \mathrm{mg} /$ day for women). ${ }^{14}$ Third, we performed regression analysis using a combined outcome of microalbuminuria and macroalbuminuria to better capture albuminuria as a continuous marker of kidney disease.

Statistical analyses were performed by SAS survey procedures (SAS version 9.1, SAS Institute, Cary, North Carolina). ${ }^{15}$ All analyses were weighted to incorporate the complex survey design of NHANES with proper reflections of stratification and clustering. Six-year weights were derived following analytic guidelines recommended by the National Center for Health Statistics. 16

\section{Results}

The study population consisted of 12,831 men and women drawn from the NHANES 1999 to 2004 after exclusion of 1,543 individuals whose urinary albumin or creatinine data were not measured, 673 individuals whose CRP data were absent, and 285 individuals with macroalbuminuria (Table 1). The study population included 52\% women, with a mean age of 46 years. Seventy-one percent were non-Hispanic white, whereas $13 \%$ and $10 \%$ were Hispanic and non-Hispanic African-American, respectively. The 1,378 participants (11\%) with microalbuminuria were more likely to be older, non-White, and of lower socioeconomic status (assessed by education and income levels) than participants without microalbuminuria. The prevalences of diabetes, current smoking, hypertension, heart failure, cerebrovascular disease, peripheral vascular disease, and anemia were significantly higher in participants with microalbuminuria compared with those without microalbuminuria. Unadjusted mean total, low-density lipoprotein, and high-density lipoprotein cholesterol levels were similar among participants with and without microalbuminuria (reflected in summary statistics), but these differences were significant after adjustment for demographic factors (reflected in $\mathrm{p}$ values).

Pearson correlations coefficient measuring how CRP and the remaining individual variables were linearly associated in our sample were low overall (all values $p<0.1$ ) (Table 2). Although correlation coefficients slightly differed depending on the computing method adopted, some common patterns were observed. CRP was positively correlated with age, triglyceride levels, and urinary albumin-to-creatinine ratio (the raw variable for microalbuminuria). In contrast, serum creatinine and high-density lipoprotein cholesterol consistently showed negative correlation with CRP concentration. 
Multivariate logistic regression modeling identified 10 predictors of prevalent microalbuminuria (age, race, education, current smoking status, history of heart failure, systolic blood pressure, diabetes mellitus, peripheral vascular disease, anemia, and CRP) among study participants (Table 3). Of the well-known traditional risk factors, diabetes, systolic blood pressure, current smoking status, and history of heart failure demonstrated the strongest associations with microalbuminuria. Of note, none of the lipid variables (i.e., triglycerides and total, low-density lipoprotein, and high-density lipoprotein cholesterol) were significant in multivariate models.

The mean CRP concentration among participants with microalbuminuria was $6.3 \mathrm{mg} / \mathrm{L}$ compared with a mean concentration of $4.0 \mathrm{mg} / \mathrm{L}$ among controls $(\mathrm{p}=0.0002)$ (Table 1$)$. On the basis of logistic regression analyses, CRP concentration was positively associated with microalbuminuria (Table 2). In the multivariate model, a $1 \mathrm{mg} / \mathrm{L}$ increase in CRP was associated with a $2 \%$ increased odds of microalbuminuria (OR 1.02, 95\% CI 1.01 to $1.02, \mathrm{p}=$ 0.0003). Nonlinear relationships assessed by squared and cubic terms of CRP were not detected.

Using categorized CRP levels (quartiles and AHA/CDC recommendations), multivariate models showed a positive trend for the association of CRP with microalbuminuria (Table 4). Multivariable-level adjustments yielded attenuated associations in all conducted analyses. Participants in the highest quartile of CRP concentration, $\geq 4.6 \mathrm{mg} / \mathrm{L}$, demonstrated a significant association with microalbuminuria (OR 1.35, 95\% CI 1.06 to $1.72, \mathrm{p}=0.017$ ) controlling for a set of traditional risk factors. According to the AHA/CDC cutpoints, compared with persons with CRP concentrations $<1 \mathrm{mg} / \mathrm{L}$, persons with CRP concentrations between 1 and $3 \mathrm{mg} / \mathrm{L}$ and $>3 \mathrm{mg} / \mathrm{L}$ were 1.15 times (95\% CI 0.94 to 1.42 ) and 1.33 times (95\% CI 1.08 to 1.65) more likely to have microalbuminuria, respectively.

The association between diabetes and microalbuminuria was the strongest of any variable in the model. We repeated analyses excluding diabetics to determine how sensitive the association between CRP and albuminuria was to the diabetic status. Comparable discrimination was observed (area under the curve $[\mathrm{AUC}]=0.71$ to 0.72 ) in all multivariate analyses, but the associations were stronger in the highest levels of the multivariate models in Table 3 (ORs increased to 1.82 and 1.57 compared with 1.35 and 1.33 , respectively). Gender-specific microalbuminuria cutpoints resulted in slightly attenuated association and discrimination (ORs of 1.28 and AUC of 0.72), but increased statistical significance (i.e., smaller $\mathrm{p} \leq 0.0006$ ) for CRP. Extending the endpoint to encompass microalbuminuria and macroalbuminuria together (omitting the corresponding exclusion criterion) yielded respective ORs of $1.36(\mathrm{p}=0.01)$ and $1.39(\mathrm{p}=0.001)$, but with increased discrimination $(\mathrm{AUC}=0.75)$.

\section{Discussion}

In this cross-sectional, population-based study, we found that CRP was significantly associated with microalbuminuria. The association was especially strong with elevated levels of CRP. Furthermore, CRP and microalbuminuria remained significantly associated after adjusting for multiple variables previously reported as determinants of microalbuminuria. The results further the hypotheses that vascular inflammation may be a determinant of microalbuminuria. The findings add evidence to the link between early renal and cardiovascular disease. Furthermore, $\mathrm{CRP}$ and urinary albumin excretion assays are inexpensive, minimally invasive, and quantitative in nature, useful attributes not only in clinical research but also, possibly, in routine patient care.

The combined 6-year data sets (1999 to 2004) of the NHANES provided a large study population with substantial representation of women, African-Americans, and Hispanics. This 
sample size, nearly twice the previously largest study of this kind, allowed for detection of a significant association between high CRP levels (i.e., $>3 \mathrm{mg} / \mathrm{L}$ ) (the currently recommended clinical cutpoint) and microalbuminuria despite the majority of participants, as expected, having CRP values well below this level. The NHANES also contained a wealth of data on potential co-morbidities (e.g., diabetes, hyperlipidemia, systolic and/or diastolic hypertension, peripheral vascular disease) that allowed for meaningful adjustments in our model. Although our findings are consistent with previous studies that have reported an association between CRP and albuminuria, ${ }^{1-7}, 9,10$ these studies have not used equally comprehensive data and, therefore, were often underpowered, not population-based, or not generalizable to the US population.

Albuminuria, an established risk marker for progressive kidney disease, has now emerged as a strong predictor of cardiovascular outcomes. ${ }^{17,18}$ Co-morbid conditions, such as diabetes and hypertension, have been proposed as pathophysiologic missing links between incipient kidney and heart disease. The demonstrated association between CRP and microalbuminuria, however, suggests that another missing link-chronic inflammation-should be considered. In addition, the emergence of CRP as a predictive biomarker for the development of cardiovascular disease has raised the intriguing question of whether this inflammatory protein is a "maker," and not just a "marker," of atherosclerosis. ${ }^{19-22}$ A similar link could be applied between vascular inflammation and the glomerular, tubular, and interstitial injuries of chronic kidney disease. Because microalbuminuria can be viewed as an early marker of endothelial dysfunction, ${ }^{23-25}$ its connection to CRP is more intuitive than glomerular filtration rate, although elevated CRP levels have also been associated with diminished creatinine clearances. 10

We have tried, in the overall model and with sensitivity analyses, to make meaningful adjustments to the data to avoid overstating the association between CRP and microalbuminuria. Nevertheless, there are certainly other factors involved with vascular inflammation, known and unknown, that we have not accounted for, and our estimate of the association between CRP and albuminuria, therefore, should be interpreted with caution. In addition, in sensitivity analysis the large representation of diabetics among the cases limited, but by no means eliminated, the validity of our results in nondiabetic populations.

Because NHANES are cross-sectional surveys, our findings only support an association between CRP and microalbuminuria and should not yield any conclusions about causation. Only a well-designed, fully powered, longitudinal study could attempt to answer the crucial questions of whether elevated CRP causes microalbuminuria, microalbuminuria causes elevated CRP levels, or, quite possibly, a third factor (or fourth, fifth, or sixth factor) leads to clinically relevant levels of both markers. Advanced glycosylation end products, hypertensioninduced endothelial damage, increased plasma cholesterol concentrations, and activation of mineralocorticoid receptors in high salt states are just a few potentially proximal factors that could cause microalbuminuria and CRP elevation.

\section{Acknowledgments}

We thank the staff and participants of the NHANES study for their important contributions and valuable resources of clinical research.

Drs. Bang, Gerber, and Mazumdar's research was partially supported by the Tolly Vinik Trust through Weill Medical College of Cornell University, New York, New York. Dr. Shoham is supported by Grant 5T-32HL007055-29 through an National Heart, Lung, and Blood Institute, Bethesda, Maryland, institutional training grant in cardiovascular disease epidemiology. 


\section{References}

1. Schalkwijk CG, Poland DC, van Dijk W, Kok A, Emeis JJ, Drager AM, Doni A, van Hinsbergh VW, Stehouwer CD. Plasma concentration of C-reactive protein is increased in type I diabetic patients without clinical macroangiopathy and correlates with markers of endothelial dysfunction: evidence for chronic inflammation. Diabetologia 1999;42:351-357. [PubMed: 10096789]

2. Yeun JY, Levine RA, Mantadilok V, Kaysen GA. C-Reactive protein predicts all-cause and cardiovascular mortality in hemodialysis patients. Am J Kidney Dis 2000;35:469-476. [PubMed: 10692273]

3. Jager A, van Hinsbergh VW, Kostense PJ, Emeis JJ, Nijpels G, Dekker JM, Heine RJ, Bouter LM, Stehouwer CD. C-reactive protein and soluble vascular cell adhesion molecule-1 are associated with elevated urinary albumin excretion but do not explain its link with cardiovascular risk. Arterioscler Thromb Vasc Biol 2002;22:593-598. [PubMed: 11950696]

4. Paisley KE, Beaman M, Tooke JE, Mohamed-Ali V, Lowe GD, Shore AC. Endothelial dysfunction and inflammation in asymptomatic proteinuria. Kidney Int 2003;63:624-633. [PubMed: 12631127]

5. Stam F, van Guldener C, Schalkwijk CG, ter Wee PM, Donker AJ, Stehouwer CD. Impaired renal function is associated with markers of endothelial dysfunction and increased inflammatory activity. Nephrol Dial Transplant 2003;18:892-898. [PubMed: 12686661]

6. Festa A, D’Agostino R, Howard G, Mykkanen L, Tracy RP, Haffner SM. Inflammation and microalbuminuria in nondiabetic and type 2 diabetic subjects: The Insulin Resistance Atherosclerosis Study. Kidney Int 2000;58:1703-1710. [PubMed: 11012904]

7. Nakamura M, Onoda T, Itai K, Ohsawa M, Satou K, Sakai T, Segawa T, Sasaki J, Tonari Y, Hiramori K, Okayama A. Association between serum C-reactive protein levels and microalbuminuria: a population-based cross-sectional study in northern Iwate, Japan. Intern Med 2004;43:919-925. [PubMed: 15575240]

8. Stuveling EM, Hillege HL, Bakker SJ, Asselbergs FW, de Jong PE, Gans RO, de Zeeuw D. C-reactive protein and microalbuminuria differ in their associations with various domains of vascular disease. Atherosclerosis 2004;172:107-114. [PubMed: 14709363]

9. Stuveling EM, Bakker SJ, Hillege HL, Burgerhof JG, de Jong PE, Gans RO, de Zeeuw D. C-reactive protein modifies the relationship between blood pressure and microalbuminuria. Hypertension 2004;43:791-796. [PubMed: 14967837]

10. Stuveling EM, Hillege HL, Bakker SJ, Gans RO, De Jong PE, De Zeeuw D. C-reactive protein is associated with renal function abnormalities in a non-diabetic population. Kidney Int 2003;63:654661. [PubMed: 12631131]

11. Wald A. Tests of statistical hypotheses concerning several parameters when the number of observations is large. Trans Am Mathematical Soc 1943;54:426-482.

12. Harrell, FE. Regression Modeling Strategies: With Applications to Linear Models, Logistic Regression, and Survival Analysis. New York: Springer; 2001.

13. Pearson TA, Mensah GA, Alexander RW, Anderson JL, Cannon RO 3rd, Criqui M, Fadl YY, Fortmann SP, Hong Y, Myers GL, et al. Markers of inflammation and cardiovascular disease: application to clinical and public health practice: a statement for healthcare professionals from the Centers for Disease Control and Prevention and the American Heart Association. Circulation 2003;107:499-511. [PubMed: 12551878]

14. Mattix HJ, Hsu CY, Shaykevich S, Curhan G. Use of the albumin/creatinine ratio to detect microalbuminuria: implications of sex and race. J Am Soc Nephrol 2002;13:1034-1039. [PubMed: 11912263]

15. An A, Watts D. New SAS procedures for analysis of sample survey data. SAS Users Group International 1998;23

16. Analytic and reporting guidelines: The National Health and Nutrition Examination Survey (NHANES). [Accessed October 23, 2007]. Available at: http://www.cdc.gov/nchs/data/nhanes/nhanes_03_04/nhanes_analytic_guidelines_dec_2005.pdf

17. de Zeeuw D, Parving HH, Henning RH. Microalbuminuria as an early marker for cardiovascular disease. J Am Soc Nephrol 2006;17:2100-2105. [PubMed: 16825327] 
18. de Zeeuw D, Remuzzi G, Parving HH, Keane WF, Zhang Z, Shahinfar S, Snapinn S, Cooper ME, Mitch WE, Brenner BM. Albuminuria, a therapeutic target for cardiovascular protection in type 2 diabetic patients with nephropathy. Circulation 2004;110:921-927. [PubMed: 15302780]

19. Paul A, Ko KW, Li L, Yechoor V, McCrory MA, Szalai AJ, Chan L. C-reactive protein accelerates the progression of atherosclerosis in apolipoprotein E-deficient mice. Circulation 2004;109:647-655. [PubMed: 14744975]

20. Verma S, Kuliszewski MA, Li SH, Szmitko PE, Zucco L, Wang CH, Badiwala MV, Mickle DA, Weisel RD, Fedak PW, Stewart DJ, Kutryk MJ. C-reactive protein attenuates endothelial progenitor cell survival, differentiation, and function: further evidence of a mechanistic link between C-reactive protein and cardiovascular disease. Circulation 2004;109:2058-2067. [PubMed: 15078802]

21. Blann AD, Lip GY. Effects of C-reactive protein on the release of von Willebrand factor, E-selectin, thrombomodulin and intercellular adhesion molecule-1 from human umbilical vein endothelial cells. Blood Coagul Fibrinolysis 2003;14:335-340. [PubMed: 12945874]

22. Nilsson J. CRP - marker or maker of cardiovascular disease? Arterioscler Thromb Vasc Biol 2005;25:1527-1528. [PubMed: 16055753]

23. Vervoort G, Wetzels JF, Lutterman JA, Bravenboer B, Berden JH, Smits P. Atrial natriuretic peptideinduced microalbuminuria is associated with endothelial dysfunction in noncomplicated type 1 diabetes patients. Am J Kidney Dis 2002;40:9-15. [PubMed: 12087555]

24. Shestakova MV, Jarek-Martynowa IR, Ivanishina NS, Kuharenko SS, Yadrihinskaya MN, Aleksandrov AA, Dedov II. Role of endothelial dysfunction in the development of cardiorenal syndrome in patients with type 1 diabetes mellitus. Diabetes Res Clin Pract 2005;68:S65-S72. [PubMed: 15955379]

25. Ochodnicky P, Henning RH, van Dokkum RP, de Zeeuw D. Microalbuminuria and endothelial dysfunction: emerging targets for primary prevention of end-organ damage. J Cardiovasc Pharmacol 2006;47:S151-S162. discussion S172-S176. [PubMed: 16794452] 
Table 1

Mean \pm SEM or prevalence $(\%)$ of risk factors by microalbuminuria case status in National Health and Nutrition Examination Surveys (NHANES) 1999 to 2004

\begin{tabular}{|c|c|c|c|}
\hline Variable & $\begin{array}{l}\text { With Microalbuminuria } \\
\qquad(\mathrm{n}=\mathbf{1 , 3 7 8})\end{array}$ & $\begin{array}{l}\text { Without Microalbuminuria } \\
\quad(\mathrm{n}=\mathbf{1 1}, \mathbf{4 5 3})\end{array}$ & p Value* \\
\hline Age (yrs) & $54.8 \pm 0.75$ & $45.1 \pm 0.29$ & $<0.0001^{*}$ \\
\hline Women & $55.5 \%$ & $52.0 \%$ & $0.01^{*}$ \\
\hline White & $67.0 \%$ & $72.7 \%$ & $0.003^{*}$ \\
\hline African-American & $12.0 \%$ & $9.9 \%$ & \\
\hline Hispanic & $14.3 \%$ & $12.9 \%$ & \\
\hline High education (>high school) & $45.0 \%$ & $54.9 \%$ & 0.0006 \\
\hline Income $(>\$ 35,000$ per year $)$ & $37.5 \%$ & $52.6 \%$ & $<0.0001$ \\
\hline Current smoker & $21.9 \%$ & $20.6 \%$ & 0.0005 \\
\hline Family history of hypertension & $25.5 \%$ & $25.9 \%$ & 0.39 \\
\hline Family history of diabetes & $35.0 \%$ & $28.6 \%$ & 0.11 \\
\hline History of cardiovascular disease & $11.4 \%$ & $4.6 \%$ & 0.007 \\
\hline History of heart failure & $5.7 \%$ & $1.7 \%$ & $<0.0001$ \\
\hline Waist (cm) & $100 \pm 0.76$ & $95.9 \pm 0.26$ & 0.008 \\
\hline Body mass index $\left(\mathrm{kg} / \mathrm{m}^{2}\right)$ & $28.9 \pm 0.30$ & $28.0 \pm 0.10$ & 0.11 \\
\hline Systolic blood pressure (mm Hg) & $135 \pm 0.86$ & $121 \pm 0.33$ & $<0.0001$ \\
\hline Diastolic blood pressure $(\mathrm{mm} \mathrm{Hg})$ & $74 \pm 0.60$ & $72 \pm 0.23$ & 0.23 \\
\hline Hypertension & $55.8 \%$ & $32.2 \%$ & $<0.0001$ \\
\hline Diabetes mellitus & $23.9 \%$ & $6.1 \%$ & $<0.0001$ \\
\hline Peripheral vascular disease & $6.9 \%$ & $2.2 \%$ & 0.002 \\
\hline Total cholesterol $(\mathrm{mg} / \mathrm{dl})$ & $203 \pm 1.8$ & $202 \pm 0.59$ & 0.02 \\
\hline HDL cholesterol (mg/dl) & $51.7 \pm 0.75$ & $52.0 \pm 0.29$ & 0.01 \\
\hline LDL cholesterol $(\mathrm{mg} / \mathrm{dl})^{\dagger}$ & $119 \pm 2.1$ & $121 \pm 0.72$ & 0.008 \\
\hline Triglycerides $(\mathrm{mg} / \mathrm{dl})^{\dagger}$ & $162 \pm 8.0$ & $142 \pm 2.2$ & 0.20 \\
\hline Dyslipidemia $^{\ddagger}$ & $9.8 \%$ & $9.9 \%$ & 0.86 \\
\hline Hemoglobin (mg/dl) & $14.3 \pm 0.08$ & $14.5 \pm 0.04$ & 0.95 \\
\hline Anemia $\$$ & $5.0 \%$ & $3.3 \%$ & 0.10 \\
\hline Serum creatinine $(\mathrm{mg} / \mathrm{dl})$ & $0.94 \pm 0.001$ & $0.88 \pm 0.003$ & 0.004 \\
\hline $\begin{array}{l}\text { Glomerular filtration rate }(\mathrm{ml} / \mathrm{min} / 1.73 \\
\left.\mathrm{m}^{2}\right)\end{array}$ & $91.2 \pm 2.9$ & $93.6 \pm 0.48$ & 0.23 \\
\hline $\mathrm{CRP}(\mathrm{mg} / \mathrm{L})$ & $6.3 \pm 0.42$ & $4.0 \pm 0.09$ & 0.0002 \\
\hline
\end{tabular}

All results were from weighted analyses to account for complex survey design.

* Unadjusted $\mathrm{p}$ values. For all other $\mathrm{p}$ values, age, race, and gender are adjusted.

${ }^{\dagger}$ Measured only on subsamples, therefore different weight was used.

*Defined as taking an antihyperlipidemic drug, fasting LDL cholesterol $\geq 160 \mathrm{mg} / \mathrm{dl}$, fasting HDL cholesterol $\leq 30 \mathrm{mg} / \mathrm{dL}$, or nonfasting calculated LDL (total cholesterol - HDL - triglycerides/5) $\geq 160 \mathrm{mg} / \mathrm{dl}$.

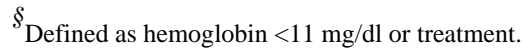


$\mathrm{HDL}=$ high-density lipoprotein LDL = low-density lipoprotein. 
Table 2

Correlation coefficients ${ }^{*}$ between C-reactive protein (CRP) and other risk factors

\begin{tabular}{llr}
\hline Variable & $\begin{array}{l}\text { Parametric } \\
\text { Pearson }\end{array}$ & $\begin{array}{r}\text { Nonparameteric } \\
\text { Spearman }\end{array}$ \\
\hline Age & 0.06 & 0.16 \\
Serum creatinine & -0.02 & -0.10 \\
Glomerular filtration rate & 0.0005 & -0.04 \\
Urinary albumin-to-creatinine ratio & 0.08 & 0.13 \\
Total cholesterol & 0.007 & 0.14 \\
High-density lipoprotein cholesterol & -0.04 & -0.13 \\
Low-density lipoprotein cholesterol & -0.01 & 0.10 \\
Triglycerides & 0.04 & 0.25 \\
\hline$*$ & &
\end{tabular}


Table 3

Multivariate logistic regression model for microalbuminuria in National Health and Nutrition Examination Surveys (NHANES) 1999 to 2004

\begin{tabular}{|c|c|c|c|c|}
\hline \multirow[t]{2}{*}{ Variable } & \multicolumn{2}{|c|}{$\begin{array}{c}\text { Demographic Factors Adjusted } \\
(\text { AUC }=0.70)\end{array}$} & \multicolumn{2}{|c|}{$\begin{array}{l}\text { Multivariables Adjusted } \\
(\mathrm{AUC}=0.75)\end{array}$} \\
\hline & OR $(95 \% \mathrm{CI})$ & p Value & OR $(95 \% \mathrm{CI})$ & p Value \\
\hline Age (yrs) & $1.04(1.03-1.04)$ & $<0.0001$ & $1.01(1.10-1.02)$ & $<0.0001$ \\
\hline Women & $1.05(0.93-1.18)$ & 0.44 & & \\
\hline White $^{\dagger}$ & $0.59(0.50-0.69)$ & $<0.0001$ & $0.73(0.62-0.87)$ & 0.0003 \\
\hline Low education ${ }^{\dagger}$ & & & $1.16(1.0-1.35)$ & 0.05 \\
\hline Current smoker & & & $1.43(1.21-1.69)$ & $<0.0001$ \\
\hline History of heart failure & & & $1.53(1.20-1.93)$ & 0.0005 \\
\hline Body mass index & & & $1.00(0.98-1.01)$ & 0.49 \\
\hline Systolic blood pressure & & & $1.02(1.02-1.03)$ & $<0.0001$ \\
\hline Diabetes mellitus & & & $3.41(2.75-4.23)$ & $<0.0001$ \\
\hline Peripheral vascular disease & & & $1.40(1.04-1.88)$ & 0.026 \\
\hline Anemia & & & $1.51(1.05-2.15)$ & 0.025 \\
\hline CRP & $1.02(1.01-1.03)$ & $<0.0001$ & $1.02(1.01-1.02)$ & 0.0003 \\
\hline
\end{tabular}

All results were from weighted analyses to account for complex survey design.

* Significant predictors or suspected confounders selected a priori are included in the final model, $\mathrm{n}=802$ participants were not included due to missing covariates.

${ }^{\dagger}$ There is no difference among African-American, Hispanic, and other races; therefore, these are combined into 1 group (reference).

${ }^{\dagger}$ Education was used instead of income for socioeconomic status because income had high missing data. 


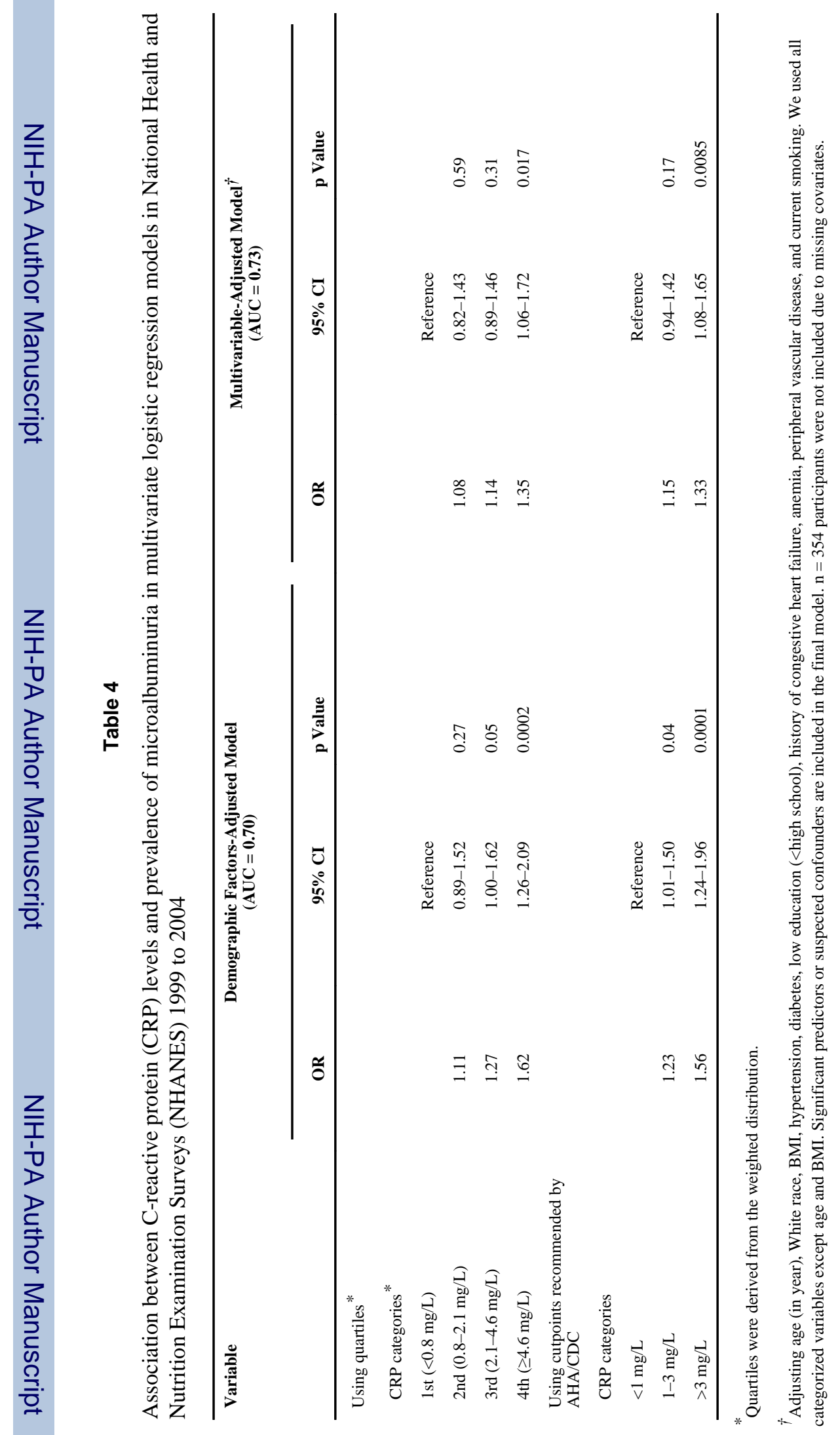

